\title{
Evaluation of the impact of warfarin time in therapeutic range on outcomes of patients with atrial fibrillation in Turkey: Perspectives from the observational, prospective WATER Registry
}

\author{
Ugur Onsel Turk ${ }^{1}$, Esref Tuncer ${ }^{1}$, Emin Alioglu$^{1}$, Kivanc Yuksel ${ }^{2}$, \\ Nihat Pekel ${ }^{3}$, Emre Ozpelit ${ }^{3}$, Ozcan Vuran $^{4}$, Istemihan Tengiz ${ }^{3}$ \\ ${ }^{1}$ Department of Cardiology, Central Hospital, Izmir, Turkey \\ ${ }^{2}$ Ege University Medicine Development and Pharmacokinetic \\ Research and Applicaton Center (ARGEFAR), Izmir, Turkey \\ ${ }^{3}$ Department of Cardiology, Izmir University, Turkey \\ ${ }^{4}$ Department of Cardiology, Ege University School of Medicine, Izmir, Turkey
}

\begin{abstract}
Background: Warfarin is highly efficacious in reducing stroke risk in patients with atrial fibrillation $(A F)$. However, its safety and efficacy in stroke prevention is markedly influenced by its time in therapeutic range (TTR). The quality of anticoagulant therapy varies considerably among countries. Representative data concerning the quality of anticoagulant therapy and its effects on clinical outcomes in Turkey are lacking.

Methods: Warfarin in Therapeutic Range (WATER) registry is a prospective, observational study which followed 572 AF patients (mean age $67.3 \pm 12$ years; females 60\%; $71 \%$ nonvalvular $A F$ ) treated with warfarin.
\end{abstract}

Results: At a median of 22-month follow-up, the mean TTR value was $42.3 \pm 18 \%$ (median: 40\%) for the whole population and lower in non-valvular AF su group than valvular AF subgroup $(40.3 \pm 18$ vs. $46.9 \pm 19$, respectively, $p<0.001)$. Death, cardiac hospitalization and minor bleeding rates were higher in the group with TTR value $<40 \%$ than the group with $>40 \%$ (3.4\% vs. $5.9 \%$; $28.6 \%$ vs. $35.4 \%$; $36.5 \%$ vs. $41.7 \%$, respectively, all of them $p<0.001)$. A correlation analysis showed a negative correlation between age and TTR value $(r=-0.178, p<0.001)$. Mean $C H A_{2} D S_{2} V A S c$ score was $3.63 \pm 1.5$ and mean HASBLED score was $2.38 \pm 1.01$ in the non-valvular AF group. A negative correlation was observed between TTR levels and $\mathrm{CHA}_{2} D S_{2} V A S c$ score.

Conclusions: WATER provides insight into the anticoagulation control status of AF patients in Turkey. The quality of anticoagulation was poor. Strategies should be undertaken by clinicians and patients to improve TTR. New oral anticoagulant agents may be perfect alternatives for non-valvular AF patients. (Cardiol J 2015; 22, 5: 567-575)

Key words: atrial fibrillation, warfarin, international normalized ratio, registry

Address for correspondence: Ugur Onsel Turk, MD, Central Hospital, Department of Cardiology, Izmir, Turkey, tel: +90 2323416767 , fax: +90 232341 6868, e-mail: droturk@yahoo.com 


\section{Introduction}

Atrial fibrillation (AF) is a common cardiac arrhythmia that is predominantly non-valvular (NVAF) and its prevalence is likely to markedly increase in next several decades [1]. AF is a common cause of stroke, heart failure, hospitalization, and death in affected patients. AF patients have a 5 -fold increase in the risk of stroke compared to individuals of the same age who are in sinus rhythm $[1,2]$. For decades, warfarin has been the most widely utilized anticoagulant for stroke prophylaxis among AF patients. Warfarin is highly efficacious in reducing stroke risk, with a meta-analysis of several clinical trials reporting a $64 \%$ decrease in stroke risk among NVAF patients treated with warfarin $[2,3]$. The major impediment to warfarin usage is the unpredictability of the level of anticoagulation in a given dose. Efficacy and safety of warfarin is markedly influenced by its time in therapeutic range (TTR), referring to the time patients treated with warfarin spend having an international normalized prothrombin time ratio (INR) within the therapeutic range, which requires regular blood test monitoring $[4,5]$. A high TTR $(\geq 70 \%)$ is required for warfarin therapy to achieve a maximal effect. This reduces the risk of not only stroke and systemic embolism, but also bleeding [3-6]. Results from randomized clinical trials demonstrate that optimal TTR threshold is reached in approximately $60 \%$ of cases [7]. However, several observational studies and registries conducted on $\mathrm{AF}$ populations reported that $\mathrm{AF}$ patients spend only approximately half the TTR of warfarin $[5,8]$. Lower TTR values in real life patients rather than randomized controlled trial patients have been confirmed consistently throughout different countries $[8,9]$. Data on AF epidemiology and management in Turkey are quite limited and prospective mild- to long-term studies are lacking. In view of this fact, we undertook the registry of Warfarin in Therapeutic Range (WATER), a prospective observational study of AF in Turkey that enrolled consecutive AF patients who received warfarin. The purposes of this registry are to observe epidemiological and clinical characteristics of the AF population and to demonstrate the quality of anticoagulant therapy with warfarin and its effects on clinical outcomes.

\section{Methods}

\section{Study design and study population}

The WATER registry was designed as a multicenter, prospective, observational study with a baseline visit at the time of patient enrolment. Between September 2011 and January 2014 we enrolled AF patients undergoing warfarin therapy for $\geq 6$ months, who had been followed up and visited at an outpatient clinic by each institution. Patients were included if they were at least 18 years old and gave written informed consent for participation in the registry. The exclusion criteria were restricted to achieve a cohort close to real life. Furthermore, consecutive patients were included at each site in order to reduce selection bias. Only patients who did not give their informed consent and who did not comply with continuous warfarin therapy were excluded. Continuous warfarin therapy was defined as warfarin use without gaps exceeding 2 months. The warfarin dose during the follow-up was adjusted to the therapeutic range proposed by the 2010 European Society of Cardiology (ESC) guidelines for the management of AF. All data were captured through an electronic case report form (eCRF). The first 7 days after treatment had started or restarted, time after permanent discontinuation of warfarin and time $>5$ days from temporary discontinuation were not included in the calculation of TTR. Periprocedural (anticoagulation bridging) INR and/or daily INR values during hospitalization were also excluded. Patients on home-monitoring or home-management were not included in the registry. Follow-ups were performed by the attending cardiologist with an office interview during INR control visits to assess the occurrence of clinical outcomes and events, as well as patients' compliance with warfarin therapy. The registry was performed according to the ethical principles of medical research involving human subjects, as specified in the Declaration of Helsinki.

\section{Definitions}

Event and status definitions were clearly defined on the eCRFs in order to achieve consistency among participating centers. TTR definition was described above. The estimation of TTR was based on the INR ranges that have been defined for individual patient's needs and clinical rationales (i.e. INR 2-3 for NVAF patients, INR 2.5-3.5 for valvular AF [VAF] patients with mitral prosthetic valve). Stroke was defined as a sudden onset of a focal neurologic deficit in a location consistent with the territory of a major cerebral artery. An event matching this definition but lasting less than $24 \mathrm{~h}$ was considered a transient ischemic attack. Intracranial hemorrhage consisted of hemorrhagic stroke and subdural or subarachnoid hemorrhage. Systemic embolism was defined as abrupt vascular 
Table 1. Demographic and clinical characteristics of the study population.

\begin{tabular}{lcccc}
\hline & $\begin{array}{c}\text { All patients } \\
(\mathbf{n}=572)\end{array}$ & $\begin{array}{c}\text { VAF } \\
\text { (n = 169; 29.5\%) }\end{array}$ & $\begin{array}{c}\text { NVAF } \\
\text { (n = 403; 70.5\%) }\end{array}$ \\
\hline Age [years] & $67.28 \pm 12.4$ & $61.44 \pm 13$ & $69.91 \pm 10$ & $<0.001$ \\
Sex (female) & $343(60 \%)$ & $116(69 \%)$ & $227(56 \%)$ & 0.0067 \\
Body mass index $\left[\mathrm{kg} / \mathrm{m}^{2}\right]$ & $27.77 \pm 5.2$ & $27.33 \pm 4$ & $27.96 \pm 6$ & 0.13 \\
Paroxysmal AF & $183(32 \%)$ & $24(14 \%)$ & $159(39 \%)$ & $<0.001$ \\
CHA $_{2}$ DS $_{2}$ VASc score & - & - & $3.64 \pm 1.53$ & - \\
HASBLED score & - & - & $2.38 \pm 1$ & - \\
Creatinine clearance $[\mathrm{mL} / \mathrm{min}]$ & $72.49 \pm 51.3$ & $79.76 \pm 33$ & $66.88 \pm 26$ & $<0.001$ \\
Heart failure & $202(35.3 \%)$ & $55(33 \%)$ & $147(36 \%)$ & 0.39 \\
Hypertension & $328(57.3 \%)$ & $71(42 \%)$ & $257(64 \%)$ & $<0.001$ \\
Diabetes mellitus & $129(22.5 \%)$ & $23(14 \%)$ & $106(26 \%)$ & $<0.001$ \\
Coronary artery disease & $160(28 \%)$ & $21(12 \%)$ & $139(34 \%)$ & $<0.001$ \\
Peripheral artery disease & $50(8.7 \%)$ & $5(3 \%)$ & $45(11 \%)$ & $<0.001$ \\
MI history & $68(11.8 \%)$ & $7(4 \%)$ & $61(15 \%)$ & $<0.001$ \\
CABG & $55(9.6 \%)$ & $12(7 \%)$ & $43(11 \%)$ & 0.215 \\
PCl & $32(5.5 \%)$ & $2(1 \%)$ & $30(7 \%)$ & 0.0022 \\
Stroke history & $63(11 \%)$ & $14(8 \%)$ & $49(12 \%)$ & 0.19 \\
Antiplatelet use & $84(14.7 \%)$ & $23(14 \%)$ & $61(15 \%)$ & 0.364 \\
\hline
\end{tabular}

$\mathrm{VAF}$ - valvular atrial fibrillation; NVAF — non-valvular atrial fibrillation; AF — atrial fibrillation; CABG — coronary artery bypass grafting; $\mathrm{PCl}$ - percutaneous coronary intervention

insufficiency associated with clinical or radiological evidence of arterial occlusion in the absence of another likely mechanism. Major bleeding was defined as a reduction in the hemoglobin level of at least $2 \mathrm{~g} / \mathrm{dL}$, a transfusion of at least 2 units of blood, or symptomatic bleeding in a critical area or organ. All other bleeding was considered minor. Duration of AF less than 7 days (with spontaneous return to sinus rhythm) or duration more than 7 days distinguished paroxysmal from persistent $\mathrm{AF}$. Hypertension was defined as blood pressure above $140 / 90 \mathrm{~mm} \mathrm{Hg}$, diabetes as fasting blood glucose above $125 \mathrm{mg} / \mathrm{dL}$, or the use of antihypertensive or antidiabetic drug therapy, respectively.

\section{Statistical analysis}

All variables collected in the eCRFs at baseline and all derived parameters were used in the statistical analysis. Binary, categorical, and ordinal parameters were summarized by means of absolute and percentage numbers within the various categories. Numerical data were summarized by means of standard statistics (i.e. mean, standard deviation, median, upper and lower quartile). Normally distributed continuous variables were expressed as mean ( \pm standard deviation) and compared with unpaired Student's t-test. Skewed variables were expressed as median (25-75 quartiles) and compared with the rank-sum test. Normality was assessed by the Shapiro-Wilk test. Differences between cohorts were tested for statistical significance using the $\chi^{2}$ test for categorical variables. Among-group comparisons were made using a nonparametric test (Kruskal-Wallis test). Correlation analyses were carried out using parametric Pearson product-moment and nonparametric Spearman's rank correlation test. A 2 -sided p-value of $<0.05$ was considered statistically significant. The statistical analysis was performed using SAS v. 9.2.

\section{Results}

\section{Patient characteristics}

Between September 2011 and January 2014, of 986 patients screened, 572 (age $67.28 \pm 12.4$ years, $59.96 \%$ female) met eligibility criteria and agreed to participate. Seventy percent of the study population had NVAF and $32 \%$ of the patients had paroxysmal AF. Clinical and demographic characteristics of the study population, NVAF and VAF subgroups are shown in Table 1 . The characteristics of the NVAF subgroup demonstrated older and sicker (accumulated comorbidities such as hypertension, diabetes, coronary artery disease, 
Table 2. Clinical events during follow-up.

\begin{tabular}{lcccc}
\hline & $\begin{array}{c}\text { All patients } \\
\text { (n= 572) }\end{array}$ & $\begin{array}{c}\text { VAF } \\
\text { (n= 169; 29.5\%) }\end{array}$ & $\begin{array}{c}\text { NVAF } \\
\text { (n = 403; 70.5\%) }\end{array}$ \\
\hline Follow-up duration (months-median) & $24.21 \pm 14(22)$ & $25.8 \pm 16(21)$ & $23.5 \pm 13(22)$ & 0.096 \\
TTR [\%] (median) & $42.26 \pm 18.4(40)$ & $46.89 \pm 18.9$ & $40.32 \pm 17.8$ & $<0.001$ \\
Death & $26(4.55 \%)$ & $6(3.5 \%)$ & $20(5 \%)$ & 0.52 \\
Stroke/TIA & $31(5.4 \%)$ & $7(4 \%)$ & $24(6 \%)$ & 0.43 \\
Intracranial bleeding & $2(0.35 \%)$ & $0(0 \%)$ & $2(0.5 \%)$ & 1 \\
Major bleeding & $29(5.1 \%)$ & $6(3.5 \%)$ & $23(5.7 \%)$ & 0.4 \\
Minor bleeding & $222(38.8 \%)$ & $64(38 \%)$ & $158(39 \%)$ & 0.78 \\
Cardiac hospitalization & $181(31.6 \%)$ & $53(31 \%)$ & $128(32 \%)$ & 1 \\
\hline
\end{tabular}

$\mathrm{VAF}$ - valvular atrial fibrillation; NVAF — non-valvular atrial fibrillation; TTR - time in therapeutic range; TIA — transient ischemic attack

Table 3. Clinical events according to median time in therapeutic range (TTR) level.

\begin{tabular}{lccc}
\hline & TTR $\geq \mathbf{4 0} \%(\mathrm{n}=\mathbf{3 1 8} ; \mathbf{5 6 \%})$ & TTR $<\mathbf{4 0 \%}(\mathrm{n}=\mathbf{2 5 4 ;} \mathbf{4 4 \% )}$ & $\mathrm{P}$ \\
\hline Death & $11(3.5 \%)$ & $15(5.9 \%)$ & 0.0003 \\
Stroke/TIA & $18(5.7 \%)$ & $13(5.1 \%)$ & 0.14 \\
Intracranial bleeding & $2(0.6 \%)$ & $0(0 \%)$ & 1 \\
Major bleeding & $18(5.7 \%)$ & $11(4.3 \%)$ & 0.085 \\
Minor bleeding & $116(36.5 \%)$ & $106(41.7 \%)$ & $<0.0001$ \\
Cardiac hospitalization & $91(28.6 \%)$ & $90(35.4 \%)$ & $<0.0001$ \\
\hline
\end{tabular}

TIA - transient ischemic attack

reduced renal function etc.) patients than the VAF subgroup.

\section{Quality of warfarin treatment and outcomes}

TTR was calculated for all patients and separately for subgroups. The mean follow-up time was $46.9 \pm 19$ (median 22) months. During the follow-up period, the mean TTR for all patients on the WATER registry was $42.26 \%$ (median $40 \%$ ). TTR value of NVAF and VAF subgroups was $40.3 \%$ and $46.9 \%$, respectively $(\mathrm{p}<0.001)$. In terms of outcome events, there was no significant difference between the subgroups (Table 2). The study population was divided into two groups according to the median TTR level. Outcome event ratios of these groups are presented in Table 3 . The TTR value $\geq 40 \%$ group had lower death, minor bleeding and cardiac hospitalization ratios than the TTR value $<40 \%$ group. A correlation analysis showed a negative correlation between TTR value and age in the whole study population $(r=-0.178, \mathrm{p}<0.001)$ (Fig. 1$)$.

\section{$\mathrm{CHA}_{2} \mathrm{DS}_{2}$ VASc score and outcomes}

Stroke risk was high in the NVAF subgroup (mean $\mathrm{CHA}_{2} \mathrm{DS}_{2}$-VASc score of $3.6 \pm 1.5$ ). The NVAF group was dissected according to
$\mathrm{CHA}_{2} \mathrm{DS}_{2} \mathrm{VASc}$ score and TTR values of these groups are presented in Figure 2. The median value of $\mathrm{CHA}_{2} \mathrm{DS}_{2} \mathrm{VASc}$ score was 4 in NVAF patients. The $\mathrm{CHA}_{2} \mathrm{DS}_{2} \mathrm{VASc}<4$ group had lower death, minor bleeding and cardiac hospitalization rates than the $\mathrm{CHA}_{2} \mathrm{DS}_{2} \mathrm{VASc} \geq 4$ group. They also had lower stroke and major bleeding rates than the $\mathrm{CHA}_{2} \mathrm{DS}_{2} \mathrm{VASc} \geq 4$ group but the differences were not statistically significant (Table 4). TTR value of $\mathrm{CHA}_{2} \mathrm{DS}_{2} \mathrm{VASc} \geq 4$ group was lower than the one of $\mathrm{CHA}_{2} \mathrm{DS}_{2} \mathrm{VASc}<4$ group and there was a negative correlation between the $\mathrm{CHA}_{2} \mathrm{DS}_{2} \mathrm{VASc}$ score and TTR value $(\mathrm{r}=-0.155, \mathrm{p}=0.018)$.

\section{HASBLED score and bleeding events}

The mean HASBLED score of the NVAF population was $2.38 \pm 1$ (median 3 ). Major bleeding events were observed in 23 cases in the NVAF group. They had a higher HASBLED score than the patients without major bleeding, however the difference was not statistically significant $(2.73 \pm 1$ vs. $2.36 \pm 1, \mathrm{p}=0.09$ ). Minor bleeding events were observed in 158 cases in the NVAF group and they had a significantly higher HASBLED score than the cases without minor bleeding events $(2.77 \pm 0.9$ vs. $2.12 \pm 1, \mathrm{p}<0.0001)$. 


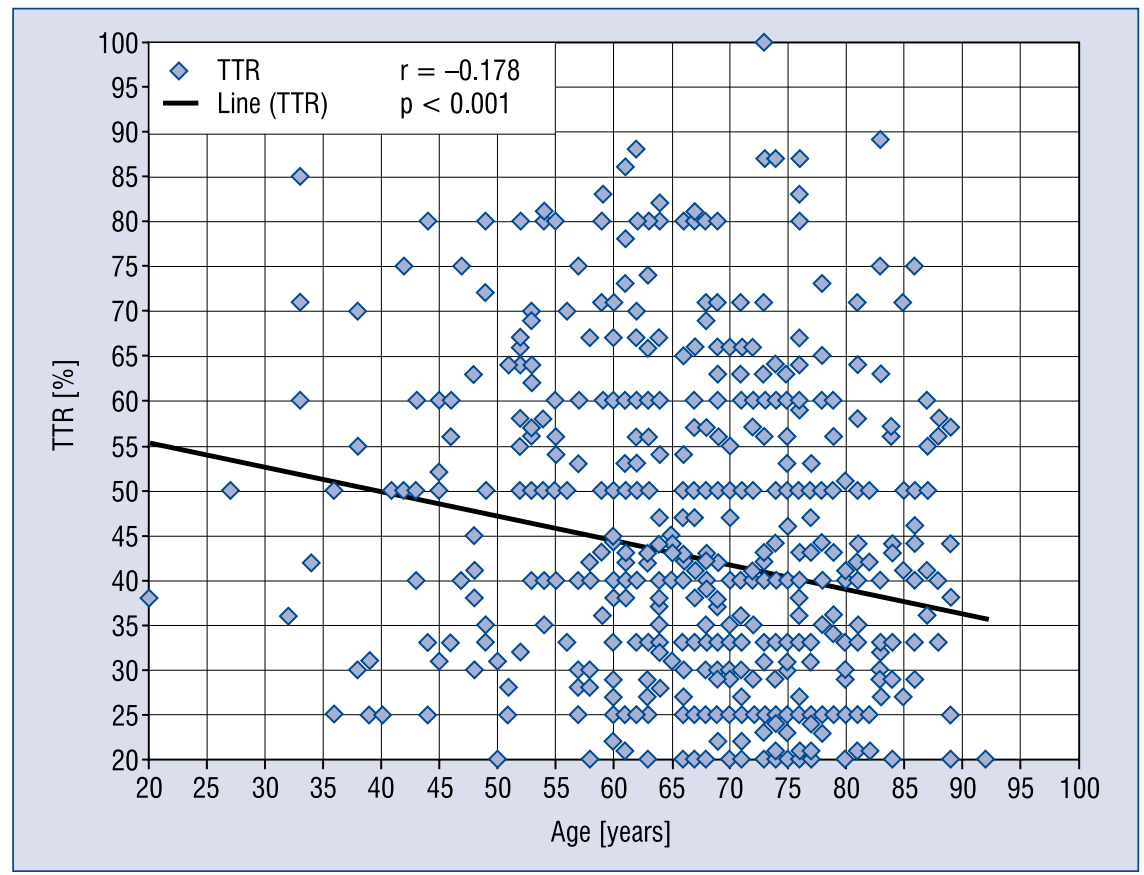

Figure 1. Correlation between age and time in therapeutic range (TTR) level.

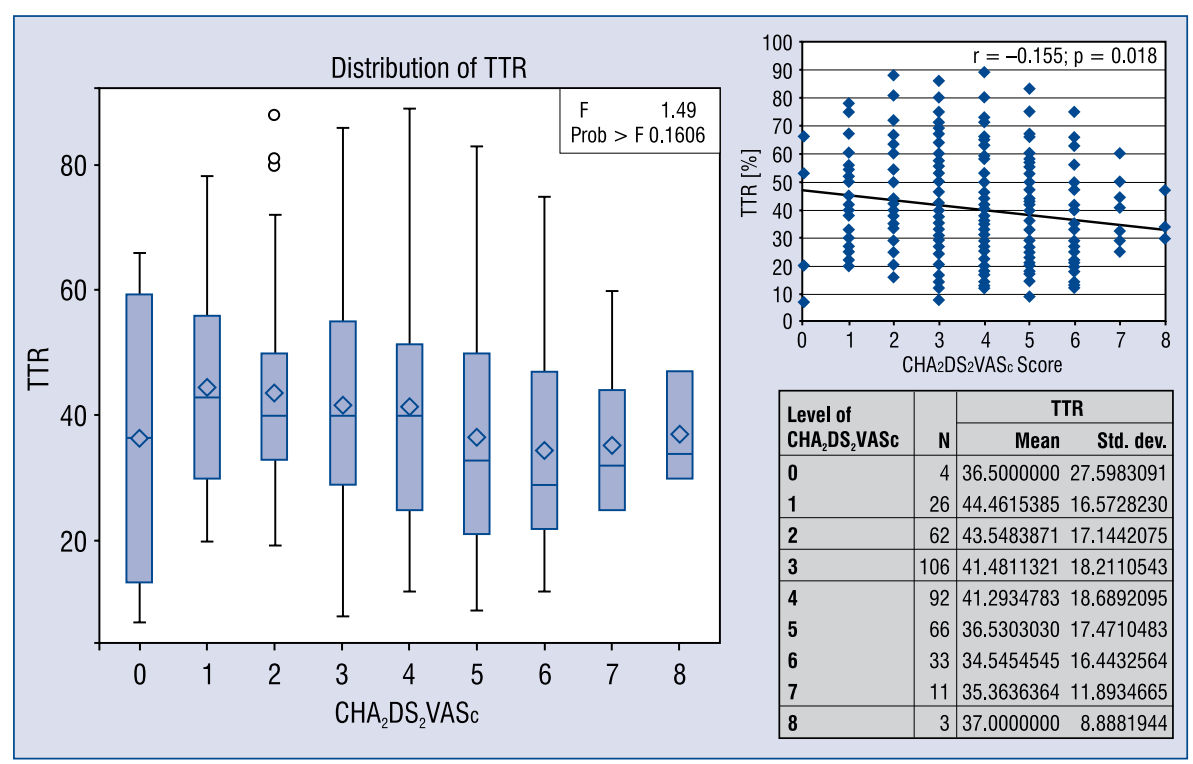

Figure 2. Distribution of time in therapeutic range (TTR) levels according to $\mathrm{CHA}_{2} \mathrm{DS}_{2} \mathrm{VASc}$ score.

\section{Discussion}

$\mathrm{AF}$ is the most common chronic rhythm disorder. It is present in over 4 million patients in Europe with a prevalence of $5-15 \%$ in patients over 80 years of age $[1,10]$. Since AF carries certain morbidity, with ischemic stroke and other embolisms, during the last decade particular atten- tion has been paid to the management of patients with AF. Several guidelines have been issued and proper anticoagulation treatment has been recommended $[2,11,12]$. However, a large percentage of AF patients receive suboptimal care. Few studies have been conducted with similar prevalence and incidence ratios in the Turkish population $[13,14]$. Notwithstanding this fact, a cohort study pointed 
Table 4. Baseline renal function, time in therapeutic range levels and clinical events according to median $\mathrm{CHA}_{2} \mathrm{DS}_{2} \mathrm{VASc}$ score.

\begin{tabular}{lccc}
\hline $\mathrm{CHA}_{2} \mathrm{DS}_{2}$ VASc score & $\geq \mathbf{4}(\mathbf{n}=\mathbf{2 0 5})$ & $<\mathbf{4}(\mathbf{n}=\mathbf{1 9 8 )}$ & $\mathrm{P}$ \\
\hline Creatinine clearance $[\mathrm{mL} / \mathrm{dk}]$ & $57.2 \pm 24.3$ & $76.9 \pm 24.3$ & $<0.001$ \\
TTR value [\%] & $38.3 \pm 17.6$ & $42.4 \pm 17.8$ & 0.019 \\
Death & $19(9 \%)$ & $1(0.5 \%)$ & $<0.001$ \\
Stroke/TIA & $16(8 \%)$ & $8(4 \%)$ & 0.14 \\
Intracranial bleeding & 1 & 1 & 1 \\
Major bleeding & $16(7.8 \%)$ & $7(3.5 \%)$ & 0.085 \\
Minor bleeding & $116(57 \%)$ & $42(21 \%)$ & $<0.001$ \\
Cardiac hospitalization & $98(48 \%)$ & $30(15 \%)$ & $<0.001$ \\
\hline
\end{tabular}

TTR — time in therapeutic range; TIA — transient ischemic attack

out that only $42 \%$ of eligible patients received oral anticoagulants [13]. Warfarin is a common anticoagulation strategy in Turkish patients with AF. However, there are no prospective, real life data about quality of anticoagulation control, factors which influence TTR values, the relationship between TTR value and clinical outcomes.

Registries are very important as means of identifying possible gaps between recommended therapies and actual everyday practice. National registries are always needed, since each country has specific features and data from other countries that may not apply elsewhere.

This registry aims to answer these questions in the Turkish population and exhibits unique results. The demographic profile of the registry population showed that nearly $70 \%$ of the AF population had non-valvular etiology. NVAF patients represented an older and sicker population than VAF population. These demographic characteristics are compatible with the European and American registries except for a higher percentage of the VAF group in the whole AF population [10, $15,16]$. These findings are unsurprising and compatible with a higher prevalence of acute rheumatic fever and rheumatic valve disease in Turkey, and also clearly exhibited that rheumatic heart disease and its late complications are still a public health concern in our country. Female dominancy was more evident in the VAF group than the NVAF group and NVAF patients had paroxysmal AF more often but had persistent and permanent AF less often than VAF patients. These demographic and clinical characteristics were previously validated in the EUROObservational Research Program on Atrial Fibrillation Pilot survey [1].

$\mathrm{AF}$ patients have a 5 -fold increase in the risk of stroke and a 2 -fold increase in the risk of mortality compared to individuals of the same age who are in sinus rhythm $[2,17]$. The registry results show that $\mathrm{AF}$ patients have a 5 -fold increase in stroke risk and a 4-fold increase in mortality compared to the general Turkish population [18-20]. The WATER registry showed that 1 in 3 patients was hospitalized due to cardiac causes and 4 in 10 patients had a minor bleeding event at their 2-year follow-up. These results reflected the health burden of $\mathrm{AF}$ in the Turkish population. However, these results should be evaluated cautiously according to the selection bias of the registry. Although a consecutive enrolment and selection of 'representative sites' (outpatients and inpatients) was used to provide a real life data set, the WATER registry only enrolled AF patients undergoing warfarin therapy. Recent data have demonstrated that while $87 \%$ of the $\mathrm{AF}$ patients were high risk patients, only $42 \%$ of these patients were on oral anticoagulant treatment in Turkey [13]. In this context, higher stroke and mortality rates should not be a surprise in the real life setting of Turkey.

Warfarin is still the main anticoagulation strategy for stroke prevention in Turkey but optimal use of warfarin in clinical practice is challenged by its narrow therapeutic window. Anticoagulation quality assessed by TTR has been closely associated with adverse outcomes, i.e., stroke, hemorrhage and mortality. In this registry, TTR levels were calculated on the basis of the INR ranges that have been defined for individual characteristics rather than simply assuming that the target range is 2.0-3.0. Although the VAF group had higher TTR values than NVAF, the registry demonstrated much poorer anticoagulation control in real life settings than similar European registries $[10,16]$. As a result, patients were spending most of their time outside of the recommended therapeutic range. The effects 
of TTR on clinical outcomes were also confirmed in the registry.

Albeit a very low mean TTR level, the study population was divided into two groups according to median TTR of $40 \%$. The lower TTR group had higher death, minor bleeding, and cardiac hospitalization rates. Although major bleeding and stroke rates were similar between the two groups, lower event rates may have had a role in these results. The quality of anticoagulation was poorer in the older population and a negative correlation was evident between age and TTR level. These results have conflicted with previous similar registries. Witt et al. [21] identified that the older age group (age $>70$ ) independently predicted INR stability. Similar results have been submitted by the VARIA Study which asserted that the group age $>55$ had a predicted lower TTR [22]. However, both studies issued quality of anticoagulation control in patients who received oral anticoagulation for any indication (AF, venous thromboembolism, prosthetic valve etc.). Additionally, many previous studies which focused on AF population found old age to be associated with lower TTR $[23,24]$. Possible explanations of the negative correlation between age and TTR are age related changes in drug metabolism, higher prevalence of co-morbidities in older patients, decline in cognitive function with increasing age and possible under treatment of the elderly with lower INR goals. Relationships between the older age, decreased renal function and lower TTR were also confirmed by the NVAF subgroup of WATER Registry. As an older group, NVAF patients may have lower cognitive function than the VAF group. We know that cognitive function has a main role in compliance of drug and INR visits. However, because the patients in the WATER Registry attended cardiology clinics regularly and received specialized care, we believe that lower TTR was not a consequence of a lack of strict INR control or undertreatment of older patients with lower INR targets and patients' access to healthcare.

Given the higher prevalence of co-morbidities in patients with NVAF, their potential association with lower TTR is of note. Anticoagulation control may be more challenging for patients with these co-morbidities. Nelson et al. [23] showed that heart failure, diabetes and previous strokes were associated with the greatest likelihood of lower TTR. WATER results also confirmed the effects of renal function on anticoagulation control. The group whose mean TTR value was lower than the median level had lower creatinine clearance than the group whose mean TTR value was higher than the median level $(67.4 \pm 27$ vs. $73.3 \pm 30 \mathrm{~mL} / \mathrm{min}$ respectively, $\mathrm{p}<0.001)$. Furthermore, there was a significant positive correlation between the creatinine clearance and TTR value $(\mathrm{r}=0.137, \mathrm{p}=0.001)$. We know that heart failure, chronic kidney disease and diabetes affect the drug pharmacokinetics and finally pharmacodynamics. An additional explanatory mechanism is a possible interaction between warfarin and multiple drugs administered to these patients. These co-morbidities may also decline cognitive function, which affects drug compliance.

If good anticoagulation control cannot be achieved within the usual care setting, specialized anticoagulation management such as anticoagulation clinics or handheld patient INR meters are validated alternative options [5, 25]. Finally, substitution with newer oral anticoagulants is inevitable in patients with NVAF.

WATER results also proved that patients with a higher $\mathrm{CHA}_{2} \mathrm{DS}_{2}$ VASc score were more likely to have a lower TTR level in the NVAF subgroup. We may hypothesize that factors contributing to the score have a major impact on drug metabolism, cognitive function and finally INR stability. Although the score does not incorporate renal function, incorporated co-morbidities such as age, hypertension, diabetes, and vascular disease could be associated with concomitant chronic kidney disease, which is known to reduce anticoagulation stability. $[5,22-24]$. The registry results confirmed the deterministic and prognostic role of $\mathrm{CHA}_{2} \mathrm{DS}_{2} \mathrm{VASc}$ score. Lower death, minor bleeding and cardiac hospitalization rates were observed in the group whose $\mathrm{CHA}_{2} \mathrm{DS}_{2} \mathrm{VASc}$ score, was higher than the median value. However there were no statistically significant differences between these groups in terms of major bleeding and stroke rates. Lower event rates, particularly major bleeding rates, may help assess the objectivity of these results. Additional dissecting the groups for higher than median value of $\mathrm{CHA}_{2} \mathrm{DS}_{2}$ VASc score may result in the inadvertent, incorrect classification of many patients who had a higher risk of stroke as lower risk patients.

The value of the HASBLED score was also confirmed with the higher score of patients who had a minor bleeding event during the follow-up. Similarly with $\mathrm{CHA}_{2} \mathrm{DS}_{2}$ VASc score, patients who had major bleeding had a statistically insignificant trend for higher HASBLED scores. As mentioned above, lower event rates may preclude reaching statistically significant differences. 


\section{Limitations of the study}

The study findings should be interpreted in the light of some limitations. Main limitations include the observational design; relatively small sample size, lower outcome rates (i.e. major bleeding, intracranial bleeding etc.) and limited 2 -year followup. Because the registry included only 3 centers from the same city, results may not be reflective of the whole country and should not be generalized. As such, we cannot claim that our findings are clearly representative of warfarin management in other centers. Certainly a larger study conducted in more centers over different geographical regions would be desirable. Moreover, some of the data wre self-reported and therefore might not have been accurate. We could not assess scheduled interruptions of oral anticoagulants (i.e. periprocedural bridging), which may have resulted in the underestimation of the TTR levels. Finally, because TTR values do not capture the full amplitude of INR fluctuations (either outside or within the therapeutic window), the TTR is a relatively crude measure of anticoagulation control. Lind et al. [26] showed that standard deviation of transformed INR is a better predictor of mortality, stroke, bleeding and hospitalization than the TTR in patients with $\mathrm{AF}$ receiving warfarin therapy. This approach may explain the difference of event ratios between the patients who had similar demographic profiles and TTR levels in WATER population.

\section{Conclusions}

In conclusion, the quality of anticoagulation with warfarin in daily clinical practice in Turkey is poor. This could have serious implications, as it is expected to adversely affect patient outcomes. These findings have implications for health policy. Strategies should be undertaken by the clinicians to improve TTR. Educational programs directed at patients are urgently needed to improve the quality of anticoagulation in Turkey. Frequent, even weekly, dose adjustments for the majority of INRs out of range is a simple concept that is associated with improved TTR and clinical outcome [27]. Systems that implement algorithm based dosing for AF patients on warfarin or widespread use of point of care testing devices for the measurement of INR may improve the management of patients [27-29]. If good anticoagulation control cannot be achieved with warfarin, new oral anticoagulants are inevitable strategies in patients with NVAF. Nevertheless, regarding the higher prevalence of valvular AF in Turkey than Europe and the United
States, strategies should not be overlooked to improve TTR in these patients.

\section{Conflict of interest: None declared}

\section{References}

1. Lip GY, Laroche C, Dan GA et al. A prospective survey in European Society of Cardiology member countries of atrial fibrillation management: Baseline results of EURObservational Research Programme Atrial Fibrillation (EORP-AF) Pilot General Registry. Europace, 2014; 16: 308-319.

2. Camm AJ, Kirchhof P, Lip GY et al. Guidelines for the management of atrial fibrillation: The Task Force for the Management of Atrial Fibrillation of the European Society of Cardiology (ESC). Europace, 2010; 12: 1360-1420.

3. Hart RG, Pearce LA, Aguilar MI. Metaanalysis: antithrombotic therapy to prevent stroke in patients who have nonvalvular atrial fibrillation. Ann Intern Med, 2007; 146: 857-867.

4. White HD, Gruber M, Feyzi J et al. Comparison of outcomes among patients randomized to warfarin therapy according to anticoagulant control: results from SPORTIF III and V. Arch Intern Med, 2007; 167: 239-245.

5. Baker WL, Cios DA, Sander SD, Coleman CI. Meta-analysis to assess the quality of warfarin control in atrial fibrillation patients in the United States. J Manag Care Pharm, 2009; 15: 244-252.

6. Oake N, Jennings A, Forster AJ, Fergusson D, Doucette S, van Walraven C. Anticoagulation intensity and outcomes among patients prescribed oral anticoagulant therapy: A systematic review and meta-analysis. CMAJ, 2008; 179: 235-244.

7. Connolly S, Pogue J, Hart R et al. Clopidogrel plus aspirin versus oral anticoagulation for atrial fibrillation in the Atrial fibrillation Clopidogrel Trial with Irbesartan for prevention of Vascular Events (ACTIVE W): A randomised controlled trial. Lancet, 2006; 367: 1903-1912.

8. van Walraven C, Jennings A, Oake N, Fergusson D, Forster AJ. Effect of study setting on anticoagulation control. A systematic review and metaregression. Chest, 2006; 129: 1155-1166.

9. Molteni M, Cimminiello C. Warfarin and atrial fibrillation: from ideal to real the warfarin affaire. Thrombosis J, 2014; 12: 5-13.

10. Kakkar AK, Mueller I, Bassand JP et al. Risk Profiles and Antithrombotic Treatment of Patients Newly Diagnosed with Atrial Fibrillation at Risk of Stroke: Perspectives from the International, Observational, Prospective GARFIELD Registry. PLoS One, 2013; 8: e63479.

11. National-Institute-for-Health-and-Care-Excellence. Atrial fibrillation: the management of atrial fibrillation 2014 (Clinical guideline 180) http://guidance.nice.org.uk/CG180.

12. January CT, Wann LS, Alpert JS et al. 2014 AHA/ACC/HRS Guideline for the Management of Patients With Atrial Fibrillation: Executive Summary: A Report of the American College of Cardiology/American Heart Association Task Force on Practice Guidelines and the Heart Rhythm Society. Circulation, 2014; 130: 2071-2104.

13. Ertas F, Eren NK, Kaya $\mathrm{H}$ et al. The atrial fibrillation in Turkey: Epidemiologic Registry (AFTER). Cardiol J, 2013; 20: 447-452.

14. Uyarel H, Onat A, Yüksel H, Can G, Ordu S, Dursunoğlu D. Incidence, prevalence, and mortality estimates for chronic atrial fibrillation in Turkish adults. Turk Kardiyol Dern Ars, 2008; 36: 214-222. 
15. Cullen MW, Kim S, Piccini JP Sr et al. Risks and benefits of anticoagulation in atrial fibrillation: Insights from the Outcomes Registry for Better Informed Treatment of Atrial Fibrillation (ORBIT-AF) registry. Circ Cardiovasc Qual Outcomes, 2013; 6: 461-469.

16. Kirchhof P, Ammentorp B, Darius H et al. Management of atrial fibrillation in seven European countries after the publication of the 2010 ESC Guidelines on atrial fibrillation: primary results of the PREvention oF thromboembolic events - European Registry in Atrial Fibrillation (PREFER in AF). Europace, 2014; 16: 6-14.

17. Kannel WB, Benjamin EJ. Current perceptions of the epidemiology of atrial fibrillation. Cardiol Clin, 2009; 27: 13-24.

18. Turkish Statistical Institute (Turkstat) website: Statistics by theme/Population and demography/vital statistics/statistical tables and dynamic search/death statistics. http://tuikapp.tuik.gov. tr/demografiapp/olum_ing.zul.

19. Ozturk S. Epidemiology of cerebrovascular diseases and risk factors-perspectives of the World and Turkey. Turkish J Geriatrics, 2009; 13: 51-58.

20. Ministry of Health. National Burden of Disease and Cost Effectiveness Study Burden of Disease Final Report, 2004 (in Turkish) Ankara: Refik Saydam Hygiene Center Presidency; 2004.

21. Witt DM, Delate T, Clark NP et al. Twelve-month outcomes and predictors of very stable INR control in prevalent warfarin users. J Thromb Haemost, 2010; 8: 744-749.

22. Rose AJ, Hylek EM, Ozonoff A, Ash AS, Reisman JI, Berlowitz DR. Patient characteristics associated with oral anticoagula- tion control: results of the Veterans AffaiRs Study to Improve Anticoagulation (VARIA) J Thrombosis Haemost, 8: 2182-2191.

23. Nelson WW, Choi JC, Vanderpoel J et al. Impact of co-morbidities and patient characteristics on international normalized ratio control over time in patients with nonvalvular atrial fibrillation. Am J Cardiol, 2013; 112: 509-512.

24. Melamed OC, Horowitz G, Elhayany A, Vinker S. Quality of anticoagulation control among patients with atrial fibrillation. Am J Manag Care, 2011; 17: 232-237.

25. O'Shea SI, Arcasoy MO, Samsa G et al. Direct-to-patient expert system and home INR monitoring improves control of oral anticoagulation. J Thromb Thrombolysis, 2008; 26: 14-21.

26. Lind M, Fahlen M, Kosiborod M, Eliasson B, Oden V. Variability of INR and its relationship with mortality, stroke, bleeding and hospitalisations in patients with atrial fibrillation. Thromb Res, 2012; 129: 32-35.

27. Hylek EM. Vitamin K antagonists and time in the therapeutic range: implications, challenges, and strategies for improvement. J Thromb Thrombolysis, 2013; 35: 333-335.

28. Okuyama Y, Matsuo M, Matsuo $\mathrm{H}$ et al. Introduction of point-ofcare testing in japanese outpatient clinics is associated with improvement in time in therapeutic range in anticoagulant-treated patients. Circ J, 2014; 78: 1342-1348.

29. DeSantis G, Hogan-Schlientz J, Liska G et al. STABLE results: warfarin home monitoring achieves excellent INR control. Am J Manag Care, 2014; 20: 202-209. 